\title{
Gold Nanoparticles for X-ray Microtomography of Neurons
}

Damien Depannemaecker, ${ }^{\dagger, \#}$ Luiz E. Canton Santos, ${ }^{\dagger}$ Antonio-Carlos Guimarães de Almeida, ${ }^{\dagger}$ Gustavo B. S. Ferreira, ${ }^{\S}$ Giovanni L. Baraldi, ${ }^{\S, \bigcirc}$ Eduardo X. Miqueles, ${ }^{\|}$Murilo de Carvalho, ${ }^{\|, \perp}$ Gabriel Schubert Ruiz Costa," Marcia J. Guimarães Marques, ${ }^{\dagger}$ Carla A. Scorza, ${ }^{\dagger}$ and Jean Rinkel*, ${ }^{\S_{\odot}}$

${ }^{\dagger}$ UNIFESP, Universidade Federal de São Paulo/Escola Paulista de Medicina (UNIFESP/EPM), São Paulo, São Paulo, Brazil

${ }^{\ddagger}$ UFSJ, Universidade Federal de São João, São João del-Rei, Minas Gerais, Brazil

${ }^{\S}$ IFGW-UNICAMP, Institute of Physics at the University of Campinas, Campinas, São Paulo, Brazil

"LNLS-CNPEM, Brazilian Synchrotron Light Laboratory, Campinas, São Paulo, Brazil

${ }^{\perp}$ LNBio-CNPEM, Brazilian Biosciences National Laboratory, Campinas, São Paulo, Brazil

ABSTRACT: Commonly used methods to visualize the biological structure of brain tissues at subcellular resolution are confocal microscopy and two-photon microscopy. Both require slicing the sample into sections of a few tens of micrometers. The recent developments in X-ray microtomography enable three-dimensional imaging at sub-micrometer and isotropic resolution with larger biological samples. In this work, we developed and compared original microtomography methods and staining protocols to improve the contrast for in vitro mouse neuron imaging. Using Golgi's method to stain neurons randomly, we imaged the whole set of mouse brain structures. For specific and nonrandom neuron labeling, we conjugated $20 \mathrm{~nm}$ gold nanoparticles to antibodies used in the immunohistochemistry (IHC) method, using anti-NeuN to label specifically neuronal nuclei. We applied an original subtraction dual-

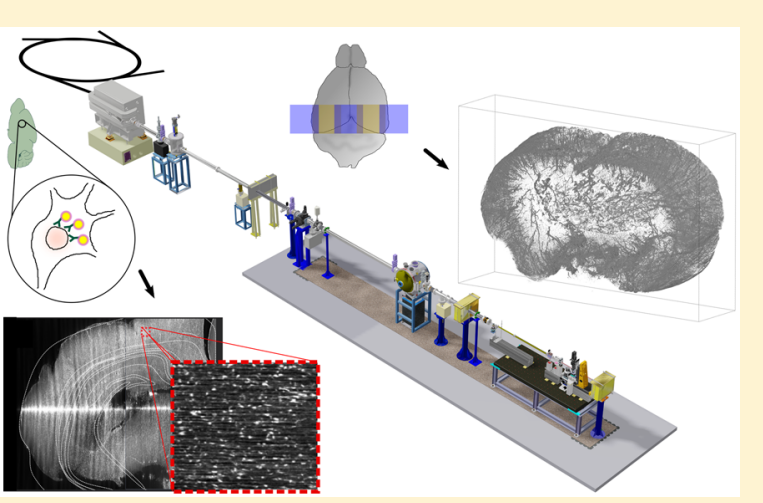
energy method for microtomography in the vicinity of the Au L-III absorption edge and compared image reconstructions to confocal microscopy images acquired on the same samples. The results show the possibility to characterize the $3 \mathrm{D}$ entire brain structure of mice. They demonstrated a high contrast and neuron detection improvement by applying the dual-energy method coupled to IHC staining.

KEYWORDS: Brain imaging, dual-energy X-ray microtomography, synchrotron, immunohistochemistry, gold nanoparticles

A challenging task in brain imaging is to integrate key information about the brain, including multiscale anatomy (macroscale, microscale, and nanoscale data collection). ${ }^{1}$ In this Letter, we present new methods to address the challenge of imaging the entire brain up to cellular and subcellular resolution. Golgi's method ${ }^{2,3}$ is a silver staining technique used to visualize neurons. This method has the disadvantage that neurons are randomly stained, but it shows suitable contrast for X-ray microtomography (or micro-CT). ${ }^{4-7}$ X-ray microCT has become a powerful technique to image biological soft tissue. ${ }^{8,9}$ However, this technique has not been fully exploited yet, specifically for nonrandom staining up to the subcellular level in brain imaging. To determine the tissue distribution of an antigen of interest, immunohistochemistry (IHC) is the most used and well-known method. Albert H. Coon developed in the early $1940 \mathrm{~s}$ a method based on the principle of antibodies binding specifically to antigens. ${ }^{10,11}$ Actually, there are many ways to visualize an antibody-antigen interaction. In light microscopy, an antibody is most commonly conjugated to an enzyme, such as peroxidase, that can catalyze a colorproducing reaction. Alternatively, the antibody can also be tagged to a fluorophore. With X-ray micro-CT, the antibody has to be tagged with a metallic particle, to produce images with sufficiently high contrast, due to photoelectric absorption. The use of gold nanoparticles for contrast in X-ray tomography has been developed during the past decade, mainly for tumors $^{12}$ and some works based on IHC-gold labeling methods. ${ }^{13}$ The innovative approach presented in this paper consists of applying X-ray micro-CT to image healthy brain tissues, with an IHC-gold labeling method.

The first objective of this experimental work is to show the possibility to characterize $3 \mathrm{D}$ whole brain structures using in vitro brain samples of mice by synchrotron micro-CT, using Golgi's staining method. The second objective is to get the distribution of one specific type of cell with the resolution of micro-CT, using a state-of-the-art IHC protocol in sliced samples. Commercially available kits for IHC are commonly used in electron microscopy studies. ${ }^{14,15}$ Thanks to such a kit, we conjugated gold nanoparticles to an antibody targeting

Received: May 16, 2019

Accepted: July 5, 2019

Published: July 5, 2019 
neuronal nuclei $(\mathrm{NeuN})$, to obtain a gold-labeled antibody conjugate.

\section{RESULTS AND DISCUSSION}

Silver Labeling. X-ray micro-CT allows the full mouse brain to be imaged, with microscopic resolution, using the panoramic acquisition technique. The reconstructed images acquired from control unstained samples did not present contrast, as shown in Figure 1a. On the contrary, the images

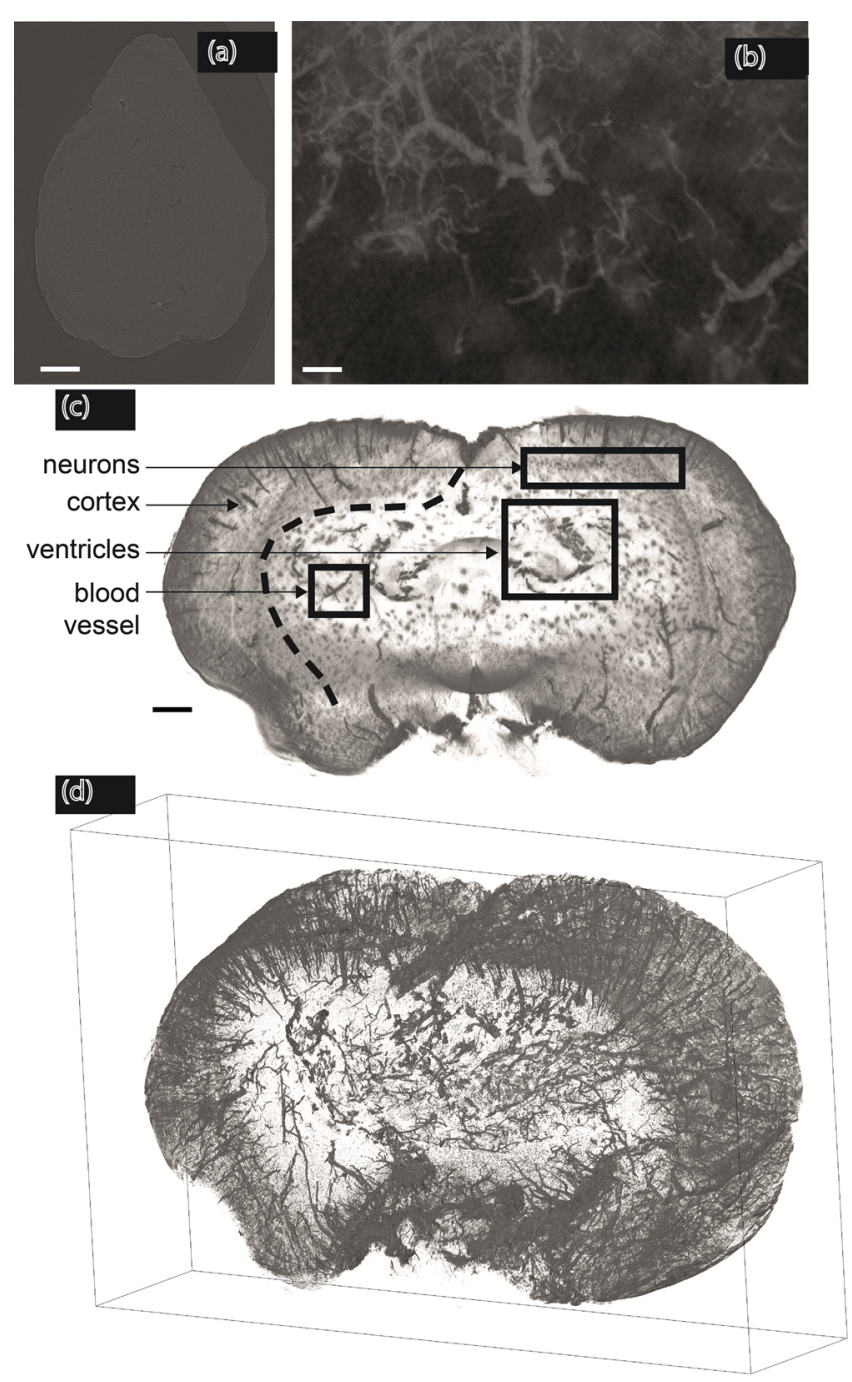

Figure 1. Golgi's micro-CT images: Single slice reconstruction of a control sample (a) (scale bar $=500 \mu \mathrm{m})$. Golgi's images: $3 \mathrm{D}$ visualization of vessels (b) (scale bar $=60 \mu \mathrm{m}$ ), projection of 600 slices in a coronal view (c) (scale bar $=500 \mu \mathrm{m})$, and 3D visualization of a subvolume of the same sample, with external box dimensions of $7.1 \mathrm{~mm}$ by $4.4 \mathrm{~mm}$ by $1.5 \mathrm{~mm}$ (d) (scale bar $=500 \mu \mathrm{m})$.

from Golgi's samples show that silver impregnated the entire brain, from peripheral to deeper regions. General structures, such as cortex, lateral ventricle, and blood vessels, appear on the reconstructed images. Figure $1 c$ shows the projection of a 3D image having a $444 \mu \mathrm{m}$ thickness, corresponding to 600 reconstructed slices with a $0.74 \mu \mathrm{m}$ slice thickness. We can observe cellular bodies of neurons, but axons and dendrite trees do not appear because of insufficient resolution and signal-to-noise ratio. Furthermore, this method allows the observation of the 3D structure of blood vessels, as shown in
Figure $1 \mathrm{~b}$ and also in the volume of Figure $1 \mathrm{~d}$. These images confirm the possibility of using X-ray micro-CT for whole brain tissue imaging, with an isotropic spatial resolution (isotropic voxel size of $0.74 \mu \mathrm{m}$ ). Nevertheless, as reported in other studies, ${ }^{16}$ the Golgi method randomly stains a few neurons, explaining the small amount of detected cellular bodies of neurons in our images. To observe the distribution of cells in a large sample and not only the morphology of a single isolated neuron, ${ }^{16}$ it is necessary to consider other staining methods, such as the IHC-gold method described in the next section.

Gold Labeling. Dual-Energy Subtraction. We labeled samples of mouse brain with NeuN antibodies conjugated to gold nanoparticles and imaged them by X-ray micro-CT and confocal microscopy. The samples are blocks of $500 \mu \mathrm{m}$ thickness. To demonstrate that the contrast is due to the gold nanoparticles, we prepared control samples with the same protocol but without conjugation to gold nanoparticles. On the high energy image of the control sample (Figure 2A), some patterns are visible but completely disappear after image subtraction (Figure 2B). This effect suggests the presence of a nonspecific contrast agent, reinforcing the interest of subtraction dual energy tomography for better structure visualization. The most probable element would be sulfur, which is part of the dimethyl sulfoxide molecule used in the postfixation process. ${ }^{17}$ It is likely that sulfur precipitates (accumulates) nonspecifically throughout the whole tissue, contributing to attenuation of the X-rays. It could also be a result of the phase contrast effect, due to sub-microscopic cracks, potentially present in dry samples. In the high energy image of the IHC sample, some structures stained by gold nanoparticles are visible (Figure 2C). Thanks to image subtraction, we can observe the contrast specifically created by the gold nanoparticles (Figure 2D).

Comparison with Microscopy. In IHC-gold samples, some structures are visible to the naked eye. It is, therefore, possible to identify structures and groups of cells from these samples in Figure 3 . In the high energy micro-CT image presented in Figure $3 \mathrm{~A}$, at Bregma $-4.8,{ }^{18}$ different structures are clearly visible, such as the cerebral cortex and the hippocampus. The high energy image contains the phase contrast effect, helping to identify some structures. ${ }^{19}$ Future works could focus on taking advantage of this phenomenon to improve structure identification. We imaged the same sample with confocal microscopy, confirming the structure identification in Figure 3B. Actually, the structures suffered separation and deformation during the drying by the critical point of $\mathrm{CO}_{2}$; also slight differences can be observed between X-ray $\mu \mathrm{CT}$ and confocal image due to slice mounting. We demonstrated the possibility to observe individual neurons by $\mathrm{X}$-ray micro-CT, as shown in Figure 3C. This identification is confirmed by the confocal microscopy observation (Figure 3D). Since the X-ray absorption is proportional to the number of gold nanoparticles, the study of isolated neurons may inform about the number of particles that stained each neuron. On the basis of this first quantification, the number of neurons could be estimated with other methods. As the method may be used with any antibody, it may permit the quantification of other types of proteins in any volume of biological tissue. The proposed protocol for Xray microtomography is promising; however, the micrometric spatial resolution is still below acceptable for cellular and subcellular analyses, especially when evaluating unknown anatomical changes. This aspect could be improved by 


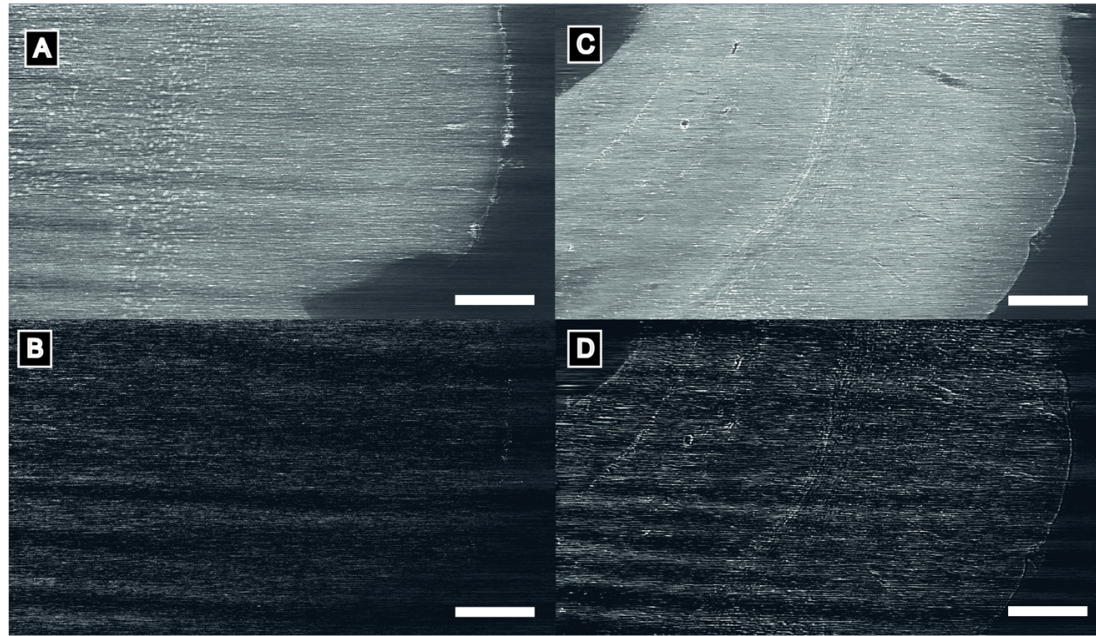

Figure 2. Effect of dual-energy subtraction. (A) Control sample high energy image, (B) control sample after dual-energy subtraction, (C) IHC-gold sample high energy image, (D) IHC-gold sample after dual-energy subtraction, scale bar $=300 \mu \mathrm{m}$.

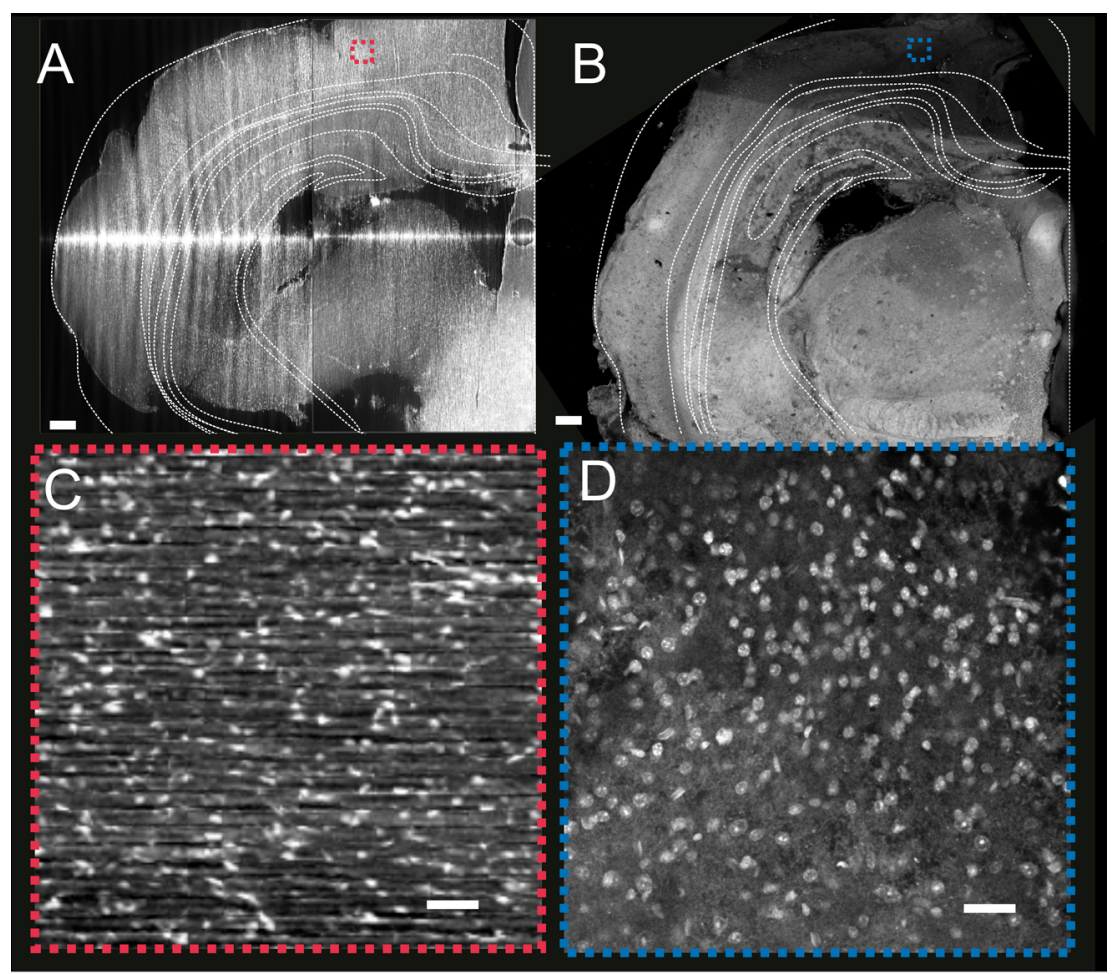

Figure 3. Mouse brain stained with the IHC-gold method. (A) X-ray micro-CT reconstruction at Bregma -4.8 , with structure identification. (B) Confocal imaging of the same sample at Bregma -4.8 , with structure identification. (A, B: White bar $=150 \mu \mathrm{m}$.) (C) Zoom into the cortical region from X-ray micro-CT. (D) Zoom into the cortical region from the confocal microscopy image. (C, D: White bar $=20 \mu \mathrm{m}$.)

combining the presented technique with phase contrast X-ray imaging (PCXI), ${ }^{19}$ for example. Finally, the reconstruction quality demonstrates the spatial stability of the samples during the data acquisition, with sub-micrometric changes. This stability is certainly promoted by the critical point of $\mathrm{CO}_{2}$ drying method, ensuring the absence of water evaporation. This aspect is associated with very low sample destruction during image acquisition, to offer an alternative to confocal microscopy with the use of specific antibodies. Future works could focus on accurately quantifying tissue and cellular structural changes after multiple beam exposures.

\section{CONCLUSION}

In this work, we demonstrate the possibility to observe neural tissue, up to the cellular resolution, without the necessity of slicing thinly. We applied two main staining techniques, based on the Golgi and IHC-gold methods. This initial work shows the significant potential of these techniques for anatomical studies of the entire brain. Results obtained with Golgi's staining offer an interesting view of the entire brain, but the nonspecific and random aspects of cell targeting by this method prevent its use for quantitative applications, such as cell counting. Our original approach consists of combining methods of IHC conjugated to nanoparticles of gold with 
(a)

(b)

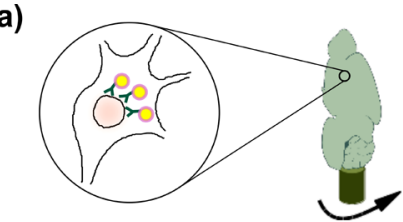

(c)
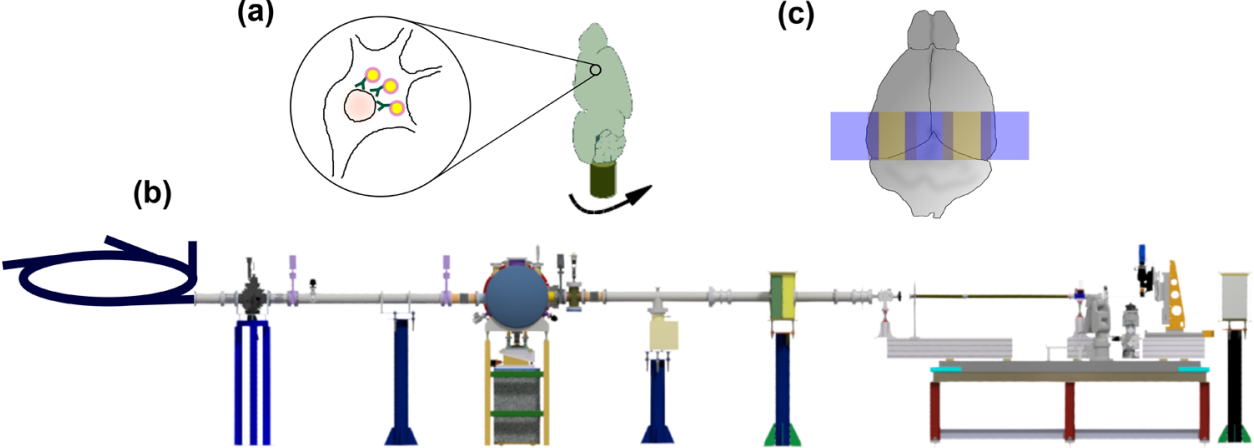

Figure 4. X-ray micro-CT acquisition. (A) Immunohistochemistry method with nano gold particles conjugated to antibody. (B) Schematic of the IMX beamline of the synchrotron. (C) Schematic of the panoramic acquisition.

synchrotron-based X-ray dual-energy subtraction micro-CT. NeuN antibodies were used to express specific target genes in neuron nuclei. Gold nanoparticles of $20 \mathrm{~nm}$ offer a sufficient contrast to neuron identification, proving that this type of particle is well suited to this imaging technique. Furthermore, image subtractions from two X-ray acquisitions at different energies allowed the specific contrast due to gold to be seen, demonstrating the possibility to visualize the distribution of specific proteins in biological tissues. This innovative approach presents a great interest over classical methods such as X-ray fluorescence and associated complexity for geometrical calibration.

\section{METHODS}

Sample Preparation. With the approval of the ethics committee of Federal University of São Paulo (approval numbers 5527120816 and 9656090218), two different protocols were applied:

Golgi Samples. Three male adult mice were anesthetized with ketamine $(90 \mathrm{mg} / \mathrm{kg})$ and xylazine $(10 \mathrm{mg} / \mathrm{kg})$ and perfused with a solution of paraformaldehyde 4\% (PFA). Brains were removed and placed for $24 \mathrm{~h}$ in a solution of PFA $4 \%$. Two brains had hemispheres separated with a razor blade. We kept one hemisphere of two brains as control samples. An entire brain and the two other hemispheres were stained with the Golgi method. Then, alternations of 3 days of immersion in a solution of $3.5 \%$ of potassium dichromate in distilled water and 3 days of immersion in a solution of $0.75 \%$ of silver nitrate in distilled water, for a total of 12 days, permit the Golgi staining. Between two immersions, we washed samples in water current for $1 \mathrm{~h}$.

IHC-Gold Samples. Two male adult mice were anesthetized with ketamine $(90 \mathrm{mg} / \mathrm{kg})$ and xylazine $(10 \mathrm{mg} / \mathrm{kg})$, and brains were removed and cut, in mouse brain matrixes with a razor blade, in coronal slices of 500 and $700 \mu \mathrm{m}$, to be stained by IHC methods. For that, we applied the protocol from the work of Kim et al. ${ }^{17}$ to selectively stain neurons with NeuN (dilution 1:50). NeuN antibodies (from Millipore company, reference MAB377) were previously conjugated to gold nanoparticles thanks to commercial conjugation kits (from abcam company, catalog reference 188215).

Dehydration and Drying. Finally, we dehydrated all samples (Golgi's and IHC-gold) by immersion in ethanol solutions with the successive concentrations 50, 60, 70, 80, 90, 95, and $100 \%$ for, at least, $1 \mathrm{~h}$ in each solution. Finally, we dried all samples with the $\mathrm{CO}_{2}$ critical point drying (CCPD) method. ${ }^{20}$ Figure $4 \mathrm{~A}$ is an overview of the method described in this work.

X-ray Microtomography. Measurements were performed at the IMX beamline (LNLS/CNPEM), with an electron source size of 391 $\mu \mathrm{m}$ by $97 \mu \mathrm{m}$ and beam divergence of $808 \mu \mathrm{rad}$ by $26 \mu \mathrm{rad}$, extracted from a $1.67 \mathrm{~T}$ bending magnet. For each brain sample, we performed three tomographic acquisitions: one with a pink beam $(5-25 \mathrm{keV}$, critical energy of $7.5 \mathrm{keV}$ ) and two with a monochromatic beam around the L-III absorption edge of gold $(11.9187 \mathrm{keV})$ at 11.85 (low energy) and $12.95 \mathrm{keV}$ (high energy), using a multilayer $\mathrm{Ru} / \mathrm{B} 4 \mathrm{C}$ monochromator. The beamline has an indirect detector for X-ray imaging, using a $50 \mu \mathrm{m}$ thickness $\mathrm{LuAg}$ scintillator and a microscope coupled with a PCO 2000 camera (2048 by 2048 pixels). Measurements used a $10 \times$ magnification objective, resulting in a pixel size of $0.74 \mu \mathrm{m}$ by $0.74 \mu \mathrm{m}$. The irradiation geometry is almost parallel, resulting in a $0.74 \mu \mathrm{m}^{3}$ voxel size. To image samples with horizontal dimension until twice the field of view $(1.52 \mathrm{~mm})$, we performed acquisition on $360^{\circ}$ for each sample, corresponding to 2048 projections with a $500 \mathrm{~ms}$ acquisition time for each projection. A schematic description is presented in Figure 4B. The panoramic frame is determined by a set of $M$ imaging cameras (frames or projections, having a size $n \times n$ with $n=2048$ ) with a minimum overlapping area, as illustrated in Figure $4 \mathrm{C}$ with $M=5$. A panoramic measurement consists of moving the sample in the $x$ direction after a prior certification that each overlapping set $O_{i j}$ is nonempty. We also note that, for a numerical absence of pitch/roll deviations, there is no need to rotate each frame for a complete data stitching. In order to maximize the signal-to-noise ratio, image reconstructions were performed using the expectation maximization (EM) iterative algorithm (40 iterations).

\section{AUTHOR INFORMATION}

\section{Corresponding Author}

*E-mail: rinkel@g.unicamp.br.

ORCID $\odot$

Jean Rinkel: 0000-0001-9980-0304

\section{Present Addresses}

${ }^{\#}$ D.D.: European Institute for Theoretical Neuroscience, CNRS, 91198 Gif sur Yvette, France.

OLNLS-CNPEM, Brazilian Synchrotron Light Laboratory, Campinas, São Paulo, Brazil.

\section{Author Contributions}

D.D., C.A.S., and J.R. conceived and designed the research project. D.D., M.J.G.M., and J.R. performed the sample preparation. D.D., G.B.S.F., G.L.B., M.d.C., G.S.R.C., and J.R. collected the data. E.X.M. performed the reconstruction of the images. D.D., L.E.C.S., A.-C.G.d.A., E.X.M., M.d.C., and J.R. performed the data analysis. D.D. and J.R. wrote the paper.

\section{Notes}

The authors declare no competing financial interest.

\section{ACKNOWLEDGMENTS}

The authors thank the IMX beamline staff (beamline proposals IMX-20170767 and IMX-20180722), the Scientific Computing Group (HPC use for image reconstruction and visualization) from LNLS, and Dra. Rita Sinigaglia Coimbra (UNIFESP) for their valuable contribution. 


\section{REFERENCES}

(1) De Felipe, J. (2015) The anatomical problem posed by brain complexity and size: a potential solution. Front. Neuroanat. 9, 104.

(2) Golgi, C. (1873) Sulla struttura della sostanza grigia dell cervello. Gazz. Med. Lomb. 33, 244-246.

(3) Ramon y Cajal, S. (1899) Textura del Sistema Nervioso del Hombre y de los Vertebrados, Moya.

(4) Westneat, M. W, and Lee, W.-K. (2008) Advances in Biological Structure, Function, and Physiology Using Synchrotron X-Ray Imaging. Annu. Rev. Physiol. 70, 119-142.

(5) Mizutani, R., Takeuchi, A., Uesugi, K., Takekoshi, S., Osamura, R. Y., and Suzuki, Y. (2008) X-ray microtomographic imaging of three-dimensional structure of soft tissues. Tissue Eng., Part C 14, 359-363.

(6) Mizutani, R., Takeuchi, A., Uesugi, K., Takekoshi, S., Osamura, R. Y., and Suzuki, Y. (2010) Microtomographic Analysis of Neuronal Circuits of Human Brain. Cerebral Cortex 20, 1739-1748.

(7) Hwu, Y., Margaritondo, G., and Chiang, A.-S. (2017) Q\&A: Why use synchrotron $\mathrm{x}$-ray tomography for multi-scale connectome mapping? BMC Biol. 15, 122.

(8) Dudak, J., Zemlicka, J., Karch, J., Patzelt, M., Mrzilkova, J., Zach, P., Hermanova, Z., Kvacek, J., and Krejci, F. (2016) High-contrast Xray micro-radiography and micro-CT of ex-vivo soft tissue murine organs utilizing ethanol fixation and large area photon-counting detector. Sci. Rep. 6, 30385.

(9) Silva, J. M. S. e., Zanette, I., Noel, P. B., Cardoso, M. B., Kimm, M. A., and Pfeiffer, F. (2015) Three-dimensional non-destructive softtissue visualization with X-ray staining micro-tomography. Sci. Rep. 5, 14088.

(10) Coons, A., Creech, H., and Jones, R. (1941) Immunological Properties of an Antibody Containing a Fluorescent Group. Exp. Biol. Med. 47, 200-202.

(11) Buchwalow, I., and Böcker, W. (2010) Immunohistochemistry: Basics and Methods, 1st ed., Springer-Verlag, Berlin, Heidelberg.

(12) Chien, C.-C., Chen, H.-H., Lai, S.-F., Wu, K.-C., Cai, X., Hwu, Y., Petibois, C., and Chu, Y. (2012) G.Margaritondo, Gold nanoparticles as high-resolution $\mathrm{X}$-ray imaging contrast agents for the analysis of tumor-related micro-vasculature. J. Nanobiotechnol. 10, 10.

(13) Popovtzer, R., Agrawal, A., Kotov, N., Popovtzer, A., Balter, J., Carey, T., and Kopelman, R. (2008) Gold nanoparticles as highresolution X-ray imaging contrast agents for the analysis of tumorrelated micro-vasculature. Nano Lett. 8, 4593-6.

(14) D'Amico, F., and Skarmoutsou, E. (2008) Quantifying immunogold labelling in transmission electron microscopy. J. Microsc. 230, 9-15.

(15) Goldberg, M. W., and Fiserova, J. (2010) Immunogold labelling for scanning electron microscopy. Methods Mol. Biol. 657, 297-313.

(16) Won Kang, H., Kyu Kim, H., Hun Moon, B., Jun Lee, S., Jung Lee, S., and Joo Rhyu, I. (2017) Comprehensive Review of Golgi Staining Methods for Nervous Tissue. Applied Microscopy 47, 63-69.

(17) Kim, S. H., Che, P., Chung, S. H., Doorn, D., Hoy, M., Larouche, M., Marzban, H., Sarna, J., Zahedi, S., and Hawkes, R. (2006) Whole-mount immunohistochemistry of the brain. Curr. Protoc Neurosci 36, 2.10.1-2.10.9.

(18) Paxinos, G., and Franklin, K. B. J. (2012) Paxinos and Franklin's the Mouse Brain in Stereotaxic Coordinates, 4th ed., Academic Press.

(19) Croton, L. C. P., Morgan, K. S., Paganin, D. M., Kerr, L. T., Wallace, M. J., Crossley, K. J., Miller, S. L., Yagi, N., Uesugi, K., Hooper, S. B., and Kitchen, M. J. (2018) In situ phase contrast X-ray brain CT. Sci. Rep. 8, 11412.

(20) Anderson, T. (1951) Techniques for the preservation of threedimensional structure in preparing specimens for the electron microscope. Trans. N. Y. Acad. Sci. 13, 130-134. 